\title{
Personalized T-cell therapy in liver transplanted patients with hepatitis $B$ virus related hepatocellular carcinoma
}

\author{
Morteza Hafezi ${ }^{1}$, Antonio Bertoletti ${ }^{1,2}$, Anthony Tan $^{1}$ \\ 'Emerging Infectious Diseases Program, Duke-NUS Medical School, Singapore 169857, Singapore. \\ ${ }^{2}$ Singapore Immunology Network, Singapore Agency for Science, Technology \& Research (A*STAR), Singapore 169857, Singapore.
}

Correspondence to: Prof. Antonio Bertoletti, Emerging Infectious Diseases Program, Duke-NUS Medical School, 8 College Road, Singapore 169857, Singapore. E-mail: antonio@duke-nus.edu.sg

How to cite this article: Hafezi M, Bertoletti A, Tan A. Personalized T-cell therapy in liver transplanted patients with hepatitis B virus related hepatocellular carcinoma. Hepatoma Res 2020;6:23. http://dx.doi.org/10.20517/2394-5079.2020.02

Received: 17 Jan 2020 First Decision: 4 Mar 2020 Revised: 18 Mar 2020 Accepted: 24 Mar 2020 Published: 11 May 2020

Science Editors: Jia Fan, Ying-Hong Shi Copy Editor: Jing-Wen Zhang Production Editor: Tian Zhang

\begin{abstract}
Hepatocellular carcinoma (HCC) is a deadly malignancy which typically occurs in the context of chronic liver inflammation. Chronic hepatitis B virus (HBV) infection is considered a major global cause of HCC development. At the moment, liver transplantation is the only curative modality for HBV-associated HCC. However, some patients develop HBV-HCC recurrence after liver transplantation, leaving them with very limited therapeutic options. Adoptive cell therapy with HBV-specific T cell receptor (TCR) that redirects T cells against HCC relapses has shown promising results in such HBV-HCC patients. In this mini-review, we discuss the application of this personalized T cell therapy, and highlight mRNA electroporation as an efficient tool for engineering safe and efficient TCR-redirected T cells for the treatment of liver transplant patients with HBV-HCC metastasis.
\end{abstract}

Keywords: HBV, TCR-T cells, mRNA, HCC metastasis, adoptive cell therapy

\section{INTRODUCTION}

Hepatocellular carcinoma (HCC) is the most common form of adult liver cancer and third leading cause of cancer-related mortality globally. Different etiological factors are involved in the pathogenesis of HCC and hepatotropic viruses like hepatitis B (HBV) and C (HCV) represent the major cause ${ }^{[1,2]}$. In regions with a high incidence of HBV (i.e., South East Asia, China, and Sub-Saharian Africa), chronic HBV infection accounts for $80 \%$ of $\mathrm{HCC}^{[3]}$. A peculiar feature of HBV-related HCC is the HBV DNA integration which

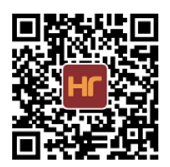


can be detected as early as 3 days post hepatocyte infection ${ }^{[3,4]}$. As such, HBV-HCC nodules can express either all or a portion of HBV antigens which can then be employed as a tumor-specific antigen to develop HBV-redirected $\mathrm{T}$ cells. We and other groups have recently developed immunotherapeutic approaches by engineering $\mathrm{T}$ cells equipped with chimeric antigen receptor $(\mathrm{CAR})^{[5]}$ or classical $\mathrm{T}$ cell receptors (TCRs) ${ }^{[6,7]}$ against HBV antigens/epitopes expressed on healthy HBV-infected hepatocytes, or in HCC cells with HBVDNA integration. Engineered TCRs or CAR redirected-T cells are able to lyse HCC cells expressing HBVspecific antigens in vitro, and showed anti-viral and anti-tumor activity in animal models ${ }^{[5,7,8]}$. In this minireview, we will discuss the use of such immunotherapies in the treatment of HBV-HCC relapses occurring post curative liver transplantation (LT). These patients will be under lifelong immunosuppressive regimens which in turn, create both obstacles and opportunities for the use of T cell therapy.

\section{LIMITATIONS OF EXISTING THERAPEUTIC MODALITIES FOR HBV-HCC RELAPSES}

Recurrent HCC after LT is deadly and difficult to manage. Treatment options include resection, radiofrequency ablation, percutaneous ethanol injection and transarterial chemoembolization in LT cases with solitary, intrahepatic HBV-associated HCC recurrence ${ }^{[9,10]}$. However, most of these HBV-HCC recurrences post-LT are systemic ${ }^{[11-14]}$, which limits treatment options. In such a scenario, currently available therapies are ineffective and first-line systemic treatment with the tyrosine kinase inhibitor, Sorafenib, can only increase survival by a few months ${ }^{[15]}$. As a new form of systemic immunotherapy, checkpoint inhibitors have shown promising outcomes in the treatment of primary $\mathrm{HCC}^{[16]}$. Checkpoint inhibitors are designed to rejuvenate immune cell function through blocking co-inhibitory receptors (e.g., PD-1, CTLA-4) expressed on exhausted immune cells. In transplant patients, this strategy can lead to undesirable and uncontrollable immune responses against the transplanted organ ${ }^{[17]}$. Indeed, attempts to use PD-1 inhibitors for the treatment of HCC relapses in LT patients led to graft rejection ${ }^{[18]}$. Therefore, at present, checkpoint inhibitors are contraindicated in LT patients with HBV-HCC.

Over the past few years, new forms of immunotherapy have been developed to specifically target HCC relapses $^{[5,7]}$. In such strategies, $\mathrm{T}$ cells are engineered to express TCRs or CARs that are able to recognize HBV epitopes in an human leukocyte antigen (HLA)-restricted manner, or detect hepatitis B $s$ antigen (HBsAg) respectively ${ }^{[6]}$. We focused our efforts on engineering TCR-redirected $\mathrm{T}$ cells as a potential treatment for $\mathrm{HBV}$-associated HCC patients. The unique features of HBV-HCC metastasis render TCR modified-T cells a better option than CAR engineered $\mathrm{T}$ cells ${ }^{[19-21]}$. In particular, HLA matching is rarely taken into consideration for liver transplantation, hence engineered TCR-T cells could only be employed to target HBV-specific antigens associated with HLA-class I molecules expressed on HCC relapses but not on the transplanted liver ${ }^{[6]}$. As a result, engineered TCR-T cells will not recognize HBV peptides present on the transplanted liver that has been re-infected by HBV and therefore, reduce the risk of possible graft rejection. Furthermore, HLA-restricted TCR-T cells would not bind to circulating soluble HBV antigens, which may instead, occur with HBV-specific CAR T cells ${ }^{[6]}$. However, in animal models, which are characterized by lower quantities of serum HBsAg than in HBV infected patients, HBsAg did not suppress the ability of CAR-T cells to recognize HBV-producing hepatocytes ${ }^{[5,19,20]}$.

\section{TREATMENT OF HBV-RELATED HCC RELAPSES WITH HBV-SPECIFIC TCR-REDIRECTED T CELLS}

The feasibility of utilizing HBV-TCR-redirected T cells for treating HBV-associated HCC was first shown in a patient who had widespread extrahepatic HBsAg+ HCC relapse ${ }^{[21]}$. In this scenario, HBV-specific TCR-T cells were engineered to recognize HBV-specific epitopes obtained from HBsAg+ HCC nodules. This patient was an ideal candidate for HBV-TCR T therapy due to multiple clinical features ${ }^{[21]}$. In this unique scenario, HBsAg was secreted exclusively by the HCC metastasis while the transplanted liver was HBsAg negative. HBsAg expression in the HCC relapses was due to integration of HBV-specific envelope DNA into the 


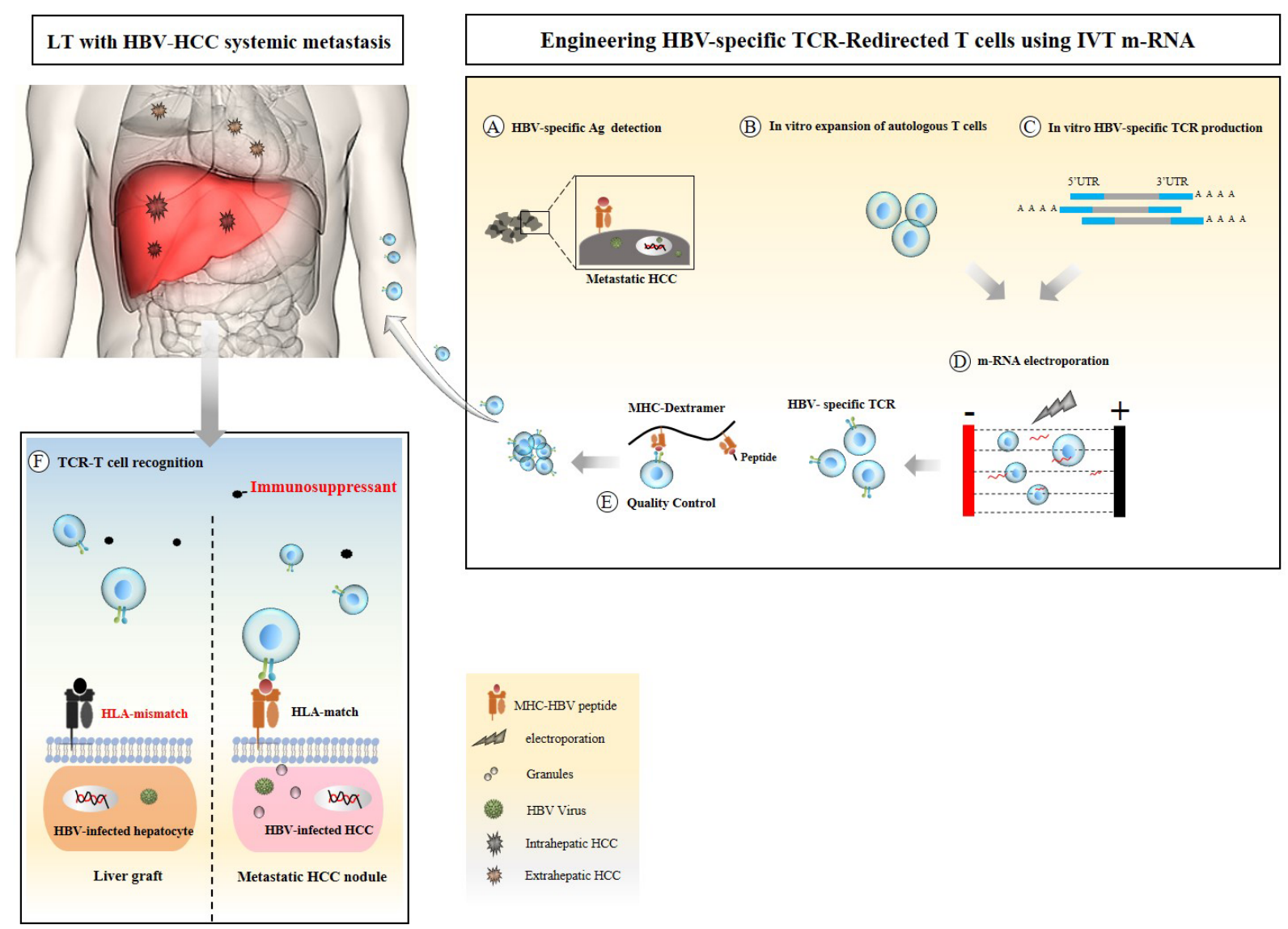

Figure 1. Schematic illustrating the development of mRNA electroporated HBV-specific TCR redirected T cells. A: identification of HBVspecific antigens expressed on metastatic tumor through molecular approaches; B: activation and expansion of autologous $T$ cells for $T$ cell engineering; C: in vitro production of mRNA encoding HBV-specific TCRs; D: developing HBV-specific TCR-redirected T cells through electroporation activated T cells with desired mRNA; E: evaluation of TCR expression and functionality of modified T cell using tetramer staining and immune assays; F: modified TCR-T cells reinfused back to LT patient to specifically target and lyse HBV-infected HCC in a HLA restricted manner with no off-tumor side effects on the HBV-infected graft. TCR: T cell receptor; HBV: hepatitis B virus; LT: liver transplantation; HCC: hepatocellular carcinoma; HLA: human leukocyte antigen; MHC: major histocompatibility complex

genome of HCC cells. Since the patient was HLA-A0201 positive, autologous T cells were engineered with a HLA-A0201-restricted TCR specific for an envelope epitope (HBs183-91). These HBV-specific TCRredirected $\mathrm{T}$ cells can specifically recognize the $\mathrm{HBsAg}+\mathrm{HCC}$ relapses, thereby reducing the risk of affecting healthy hepatocytes during treatment [Figure 1]. Using retroviral gene editing, the coding sequences of alpha and beta TCR were introduced to the T cells in vitro. Following infusion of a single dose of autologous retroviral transduced TCR-T cells $\left(\sim 2 \times 10^{4}\right.$ TCR-T cells $\left./ \mathrm{kg}\right)$, HBV-specific T cells expanded efficiently, reaching up to $\sim 2 \%$ of blood-circulating CD8 T cells and reduced serum HBsAg levels by up to $90 \%$ within 30 days post-infusion without notable side effects. Unfortunately, the patient's treatment started at a very late stage when there were already multiple metastases in the lungs, bones and neck. The patient was monitored for $\sim 8$ months post-T cell infusion and despite reduction of HBsAg levels, the patient's general medical condition subsequently declined and he succumbed to his medical complications. The reduction of serum HBsAg together with the expansion of the infused HBV-specific TCR-redirected T cells strongly suggest that HCC relapses expressing HBV antigens can be targeted by HBV-redirected T cells.

We therefore expanded our research efforts to better understand the treatability of HBV-related HCC relapses with this new form of immunotherapy. 


\section{ABILITY OF HBV-SPECIFIC TCR REDIRECTED T CELLS TO RECOGNIZE HCC CELLS WITH SHORT HBV-DNA INTEGRATION}

As described above, the feasibility of T-cell immunotherapy was first demonstrated in the scenario wherein HBsAg was expressed and secreted only by the HCC metastases and not by the transplanted liver. However, unlike that patient, HBV-DNA integrations in HCC cells do not always involve the complete open reading frame of HBV antigens. Although HBV-DNA integration could be seen in 60\%-80\% of HCC cases, most HCC cells appear HBsAg negative when tested with antibodies targeting the whole $\mathrm{HBs} \mathrm{Ag}^{[22,23]}$. This is easily explained by the detection of HBV-DNA integration that is often incomplete in normal and transformed hepatocytes and hence generate HBV-human chimeric protein ${ }^{[24-26]}$ and not whole HBV proteins.

Antibody-based techniques, which depend on the recognition of conformational epitopes, are unable to detect these chimeric proteins and therefore these HBV-HCC tumours are often negative for HBV antigens ${ }^{[22,27]}$. This explains why utilizing HBV-related antigens as a tumor-specific antigen was highly controversial and thought to be applicable only for a minority of HCC patients presenting whole HBV antigens in their HCC relapses. However, unlike antibodies, TCRs detect short linear sequences of HBV antigens (9-10 amino acid long) that can be derived from HBV-human chimeras present in HBV serologically negative HCC cells. We have recently tested this possibility in vitro utilizing HCC cells negative for HBV antigens when assayed using antibody based techniques ${ }^{[12]}$. We utilized HBV-specific CD8 T cells and antibody specific for HLA-class I/HBV epitopes and were able to demonstrate that the production and presentation of $\mathrm{HBV}$-specific CD8 $\mathrm{T}$ cell epitopes can take place in naturally HBV serologically negative HCC cells with short HBV-DNA integration ${ }^{[12]}$. This in vitro demonstration showed the possibility of utilizing short sequences of HBV-DNA integration present in the majority of HBV-related HCC as a robust tumor-associated antigen which can then be applied for HBV-specific T cell engineering. Furthermore, despite the high genetic diversity between HCC cells in the same patient ${ }^{[28-30]}$, single-cell genome sequencing of HBV-HCC cells showed remarkable homology of HBV integration across multiple single tumor cells ${ }^{[31]}$. This suggests that TCR-redirected T cells specific for single epitopes should be able to target the majority of HCC cells present in a patient.

To show feasibility of this strategy, HBV integration profiles of HCC metastases from two LT patients with undetectable HBsAg in the serum were analyzed ${ }^{[12]}$. HBV-specific TCR-redirected $\mathrm{T}$ cells targeting the epitope encoded by the detected HBV integrations were engineered. Following adoptive T cell therapy, significant volume reduction of several pulmonary metastases has been seen in one patient.

Importantly, in these patients, TCR-T cells were engineered through mRNA electroporation and not virally transduced. This approach offers inherent safety features due to the labile nature of mRNA [Figure 1]. Unlike viral DNA, mRNA transfection has no risk of causing random integration in the human genome. More importantly, controlled pharmacokinetics of the therapy allows for dose escalation by multiple repeat injections which can be personalized for each patient. Multiple infusion of mRNA TCR-T cells ( up to 600 million TCR-T cells) showed no significant increase in serum cytokine levels as well as no evidence of graft inflammation in both patients after receiving this therapy ${ }^{[12]}$. Although this therapy was not able to completely eliminate HCC relapses in both patients, one had a dramatic reduction in size of multiple metastatic lesions in the lung without the detection of new lesions and he was clinically well for almost 2 years during treatment ${ }^{[12]}$. Unfortunately, abdominal and retroperitoneal HCC relapses that were already present in the patient at the time of TCR therapy did not show any response, progressively expanded and ultimately led to the patient's death (Personal communication). Even though we were unable to investigate the real cause of the unresponsiveness of HCC relapses in different anatomical locations, such data suggests that a tumor's anatomical location together with its inflammatory status, could have a negative impact on TCR-T therapy outcome. 


\section{FUTURE DIRECTIONS IN HBV-HCC T CELL THERAPY}

Despite encouraging results from the initial trials of TCR-redirected T cell therapy in LT patients with HCC relapses, there is still a clear need for improvement of such therapies. Multiple factors present in the LT sera could have an impact on the outcome of TCR-T immunotherapy ${ }^{[32]}$. The impact of such variables also remains largely unexplored and needs to be addressed. One of the main obstacles of $\mathrm{T}$ cell therapy post-LT is the pharmacological immunosuppression started after transplantation to prevent graft rejection ${ }^{[33]}$. Longterm maintenance immunosuppression typically achieved by Tacrolimus alone, or in combination with mycophenolate mofetil continues throughout the patient's life. These drugs were designed to broadly impair $\mathrm{T}$ effector function, therefore potentially impacting the adoptively transferred HBV-specific TCR-T cells in vivo. With regard to that, in vitro experiments in a $3 \mathrm{D}$ model clearly showed that the mTOR inhibitor, Rapamycin, could impair TCR-redirected T cell migration and cytotoxicity ${ }^{[34]}$. Engineering T cells to be resistant to such drugs or using different subsets of $\mathrm{T}$ cells with inherent resistant features could allow engineered TCR-T cells to function effectively even in the face of strong immunosuppression. For instance, knock-down of FK506-binding protein 1a, tacrolimus-specific binding protein, markedly recovers $\mathrm{T}$ cell function in the presence of clinically-relevant concentrations of Tacrolimus, therefore the same strategy could be employed to develop drug-resistant TCR T cells for the treatment of LT with HBV-HCC relapses ${ }^{[35]}$. Furthermore, at this moment, only $\alpha \beta$ TCR T cells are used to engineer therapeutic HBV-specific TCR T cells. Alternatively, the desired $\alpha \beta$ TCR can also be introduced into other immune cells to provide extra benefits. For example, mucosal-associated invariant $\mathrm{T}$ cells are non-conventional T cells representing 20\%$45 \%$ of the liver $\mathrm{T}$ cell repertoire ${ }^{[36,37]}$. These cells express a high level of multi-drug resistant pump called ATP-Binding cassette subfamily b member $1(\mathrm{ABCB} 1)$ which allows mucosal-associated invariant $\mathrm{T}$ cells to retain their function in the face of chemotherapeutic intervention ${ }^{[36,38]}$. This drug-resistant feature, together with liver specificity make these cells a highly promising candidate for developing new therapeutic $\mathrm{T}$ cells for the treatment of liver associated cancers. Altogether, developing drug-resistant therapeutic TCR T cells could have the potential for clinical application in the treatment of HBV-HCC relapses after curative LT, where immunotherapeutic TCR T-cell function can still be exerted in the presence of obligate immunosuppression. In our opinion, implementing these immunosuppressive drug resistant armored -TCR T cells could improve current immunotherapy outcomes in HBV-associated HCC relapses and would most likely increase survival rates by slowing down tumor progression.

\section{DECLARATIONS}

\section{Authors' contributions}

Wrote the manuscript: Hafezi M, Bertoletti A, Tan A

\section{Availability of data and materials}

Not applicable.

\section{Financial support and sponsorship}

This work was supported by a Singapore Translational Research (STaR) investigator award [MOH-000019 (MOH_STaR17nov-001)] and by a National Research Foundation (Singapore) award (NRF-CRP17-2017-06).

\section{Conflicts of interest}

All authors declared that there are no conflicts of interest.

\section{Ethical approval and consent to participate}

Not applicable.

\section{Consent for publication}

Not applicable. 


\section{Copyright}

(c) The Author(s) 2020.

\section{REFERENCES}

1. Akinyemiju T, Abera S, Ahmed M, Alam N, Alemayohu MA, et al.; Global Burden of Disease Liver Cancer Collaboration. The burden of primary liver cancer and underlying etiologies from 1990 to 2015 at the global, regional, and national level: results from the global burden of disease study 2015. JAMA Oncol 2017;3:1683-91.

2. Ringelhan M, Pfister D, O’Connor T, Pikarsky E, Heikenwalder M. The immunology of hepatocellular carcinoma. Nat Immunol 2018;19:222-32.

3. Yang JD, Hainaut P, Gores GJ, Amadou A, Plymoth A, et al. A global view of hepatocellular carcinoma: trends, risk, prevention and management. Nat Rev Gastroenterol Hepatol 2019;16:589-604.

4. Tang KW, Alaei-Mahabadi B, Samuelsson T, Lindh M, Larsson E. The landscape of viral expression and host gene fusion and adaptation in human cancer. Nat Commun 2013;4:2513.

5. Krebs K, Bottinger N, Huang LR, Chmielewski M, Arzberger S, et al. T cells expressing a chimeric antigen receptor that binds hepatitis B virus envelope proteins control virus replication in mice. Gastroenterology 2013;145:456-65.

6. Gehring AJ, Xue SA, Ho ZZ, Teoh D, Ruedl C, et al. Engineering virus-specific T cells that target HBV infected hepatocytes and hepatocellular carcinoma cell lines. J Hepatol 2011;55:103-10.

7. Koh S, Shimasaki N, Suwanarusk R, Ho ZZ, Chia A, et al. A practical approach to immunotherapy of hepatocellular carcinoma using T cells redirected against hepatitis B virus. Mol Ther Nucleic Acids 2013;2:e114.

8. Kah J, Koh S, Volz T, Ceccarello E, Allweiss L, et al. Lymphocytes transiently expressing virus-specific T cell receptors reduce hepatitis B virus infection. J Clin Invest 2017;127:3177-88.

9. Lim HK. Radiofrequency thermal ablation of hepatocellular carcinomas. Korean J Radiol 2000;1:175-84.

10. Zhou B, Shan H, Zhu KS, Jiang ZB, Guan SH, et al. Chemoembolization with lobaplatin mixed with iodized oil for unresectable recurrent hepatocellular carcinoma after orthotopic liver transplantation. J Vasc Interv Radiol 2010;21:333-8.

11. Na GH, Hong TH, You YK, Kim DG. Clinical analysis of patients with hepatocellular carcinoma recurrence after living-donor liver transplantation. World J Gastroenterol 2016;22:5790-9.

12. Tan AT, Yang N, Lee Krishnamoorthy T, Oei V, Chua A, et al. Use of expression profiles of HBV-DNA integrated into genomes of hepatocellular carcinoma cells to select T cells for immunotherapy. Gastroenterology 2019;156:1862-76.e9.

13. Zhang HM, Shi YX, Sun LY, Zhu ZJ. Hepatocellular carcinoma recurrence in living and deceased donor liver transplantation: a systematic review and meta-analysis. Chin Med J (Engl) 2019;132:1599-609.

14. Sugawara Y. Living-donor liver transplantation for patients with hepatocellular carcinoma in Japan: current situations and challenge. Hepatobiliary Pancreat Dis Int 2020;19:1-2.

15. Llovet JM, Ricci S, Mazzaferro V, Hilgard P, Gane E, et al. Sorafenib in advanced hepatocellular carcinoma. N Engl J Med 2008;359:378-90.

16. El-Khoueiry AB, Sangro B, Yau T, Crocenzi TS, Kudo M, et al. Nivolumab in patients with advanced hepatocellular carcinoma (CheckMate 040): an open-label, non-comparative, phase 1/2 dose escalation and expansion trial. Lancet 2017;389:2492-502.

17. Gassmann D, Weiler S, Mertens JC, Reiner CS, Vrugt B, et al. Liver allograft failure after nivolumab treatment-A case report with systematic literature research. Transplant Direct 2018;4:e376.

18. Hu B, Yang XB, Sang XT. Liver graft rejection following immune checkpoint inhibitors treatment: a review. Med Oncol 2019;36:94.

19. Bertoletti A, Brunetto M, Maini MK, Bonino F, Qasim W, et al. T cell receptor-therapy in HBV-related hepatocellularcarcinoma. Oncoimmunology 2015;4:e1008354.

20. Kruse RL, Shum T, Tashiro H, Barzi M, Yi Z, et al. HBsAg-redirected T cells exhibit antiviral activity in HBV-infected human liver chimeric mice. Cytotherapy 2018;20:697-705.

21. Qasim W, Brunetto M, Gehring AJ, Xue SA, Schurich A, et al. Immunotherapy of HCC metastases with autologous T cell receptor redirected T cells, targeting HBsAg in a liver transplant patient. J Hepatol 2015;62:486-91.

22. Wang Y, Wu MC, Sham JS, Tai LS, Fang Y, et al. Different expression of hepatitis B surface antigen between hepatocellular carcinoma and its surrounding liver tissue, studied using a tissue microarray. J Pathol 2002;197:610-6.

23. Wong DKH, Cheng SCY, Mak LLY, To EWP, Lo RCL, et al. Among patients with undetectable hepatitis B surface antigen and hepatocellular carcinoma, a high proportion has integration of HBV DNA into hepatocyte DNA and no cirrhosis. Clin Gastroenterol Hepatol 2020;18:449-56.

24. Chiu YT, Wong JK, Choi SW, Sze KM, Ho DW, et al. Novel pre-mRNA splicing of intronically integrated HBV generates oncogenic chimera in hepatocellular carcinoma. J Hepatol 2016;64:1256-64.

25. Furuta M, Tanaka H, Shiraishi Y, Uchida T, Imamura M, et al. Correction: characterization of HBV integration patterns and timing in liver cancer and HBV-infected livers. Oncotarget 2018;9:31789.

26. Furuta M, Tanaka H, Shiraishi Y, Unida T, Imamura M, et al. Characterization of HBV integration patterns and timing in liver cancer and HBV-infected livers. Oncotarget 2018;9:25075-88.

27. Fu S, Li N, Zhou PC, Huang Y, Zhou RR, et al. Detection of HBV DNA and antigens in HBsAg-positive patients with primary hepatocellular carcinoma. Clin Res Hepatol Gastroenterol 2017;41:415-23.

28. Hou Y, Guo H, Cao C, Li X, Hu B, et al. Single-cell triple omics sequencing reveals genetic, epigenetic, and transcriptomic heterogeneity in hepatocellular carcinomas. Cell Res 2016;26:304-19.

29. Correction for Ling et al., extremely high genetic diversity in a single tumor points to prevalence of non-Darwinian cell evolution. Proc Natl Acad Sci U S A 2016;113:E663. 
30. Ling S, Hu Z, Yang Z, Yang F, Li Y, et al. Extremely high genetic diversity in a single tumor points to prevalence of non-Darwinian cell evolution. Proc Natl Acad Sci U S A 2015;112:E6496-505.

31. Duan M, Hao J, Cui S, Worthley DL, Zhang S, et al. Diverse modes of clonal evolution in HBV-related hepatocellular carcinoma revealed by single-cell genome sequencing. Cell Res 2018;28:359-73.

32. Hafezi M, Bertoletti A, Tan AT. T cell immunotherapy in hepatitis B virus related hepatocellular carcinoma. Hepatoma Res 2018;4:16.

33. Pillai AA, Levitsky J. Overview of immunosuppression in liver transplantation. World J Gastroenterol 2009;15:4225-33.

34. Pavesi A, Tan AT, Koh S, Chia A, Colombo M, et al. A 3D microfluidic model for preclinical evaluation of TCR-engineered T cells against solid tumors. JCI Insight 2017;2.

35. De Angelis B, Dotti G, Quintarelli C, Huye LE, Zhang L, et al. Generation of Epstein-Barr virus-specific cytotoxic T lymphocytes resistant to the immunosuppressive drug tacrolimus (FK506). Blood 2009;114:4784-91.

36. Dusseaux M, Martin E, Serriari N, Peguillet I, Premel V, et al. Human MAIT cells are xenobiotic-resistant, tissue-targeted, CD161hi IL-17-secreting T cells. Blood 2011;117:1250-9.

37. Kurioka A, Walker LJ, Klenerman P, Willberg CB. MAIT cells: new guardians of the liver. Clin Trans1 Immunol 2016;5:e98.

38. Turtle CJ, Swanson HM, Fujii N, Estey EH, Riddell SR. A distinct subset of self-renewing human memory CD8+ T cells survives cytotoxic chemotherapy. Immunity 2009;31:834-44. 